\title{
Does DNA methylation influence the effects of psychiatric drugs?
}

\author{
"While sequence polymorphisms of DNA are enduring \\ genetic factors, the extent of CPG methylation may have multiple \\ influences, both environmental (in the broadest interpretation of \\ that term) and genetic."
}

\section{First draft submitted: 2 December 2015; Accepted for publication: 8 December 2015; Published online: 26 February 2016}

Keywords: ADHD • antidepressants • antipsychotics • depression • DNA methylation - pharmacogenetics $\bullet$ schizophrenia $\bullet$ treatment response

The introduction, in the middle of the last century, of specific drug treatments for schizophrenia, bipolar illness and depression brought relief to many of those suffering from these disorders. Subsequently developed drugs have offered some improvements in tolerability, through changes in side effect profiles, but not more than marginal changes in efficacy. Thus severe mental illness, despite the undoubted value of current pharmacotherapy, still represents a huge economic and social burden, reflecting the limited efficacy of drug treatments. Treatment of depression achieves response rates of little more than $50 \%$, while even lower proportions of people with schizophrenia achieve adequate symptom relief with the antipsychotic drugs.

Thus there are profound differences between individuals with severe mental illness in how they respond to drug treatments. In addition to the wide variability in symptom response mentioned above, the experience of adverse drug effects can also vary greatly between individuals. These differences in efficacy and side effects are thought to reflect, at least in part, genetic differences between patients; thus pharmacogenetics in psychiatry has much to offer in the eventual identification of risk factors for these limitations of drug treatment.

Over the past two decades, research into the pharmacogenetics of psychiatric drugs has identified several genetic variations that reliably associate with some of the effects of drug treatment, as well as many more that provide intriguing but inadequately replicated indications of gene associations. There appears to have been more success in identifying genetic factors relating to side effects of drug treatment than in finding associations with symptom response. This may be because the phenotypes related to adverse effects, such as weight gain, are often easily defined and objectively measurable, while disease severity in psychiatry is multifactorial with complex and subjective measures.

Nevertheless, some consistent findings relating to treatment response of symptoms of depression and schizophrenia have been found, particularly when one considers neurotransmitter systems rather than individual genes or SNPs. For example, variations in genes related to serotonin (5-HT) synthesis, disposition and its receptors have variously been implicated in antidepressant response. Many of the various pharmacogenetic findings involve functional SNPs in $5^{\prime}$ regulatory regions. Often these polymorphisms are within islands of $\mathrm{CpG}$ sequences, sites for DNA methylation that contribute to the epigenetic control of transcription. Thus, it seems very plausible that the extent of $\mathrm{CpG}$ methylation of specific promoter sequences that are recognition sites for transcription factors might correlate, as SNPs can associate, with the consequences of drug treatments.

While sequence polymorphisms of DNA are enduring genetic factors, the extent of
Epigenomics
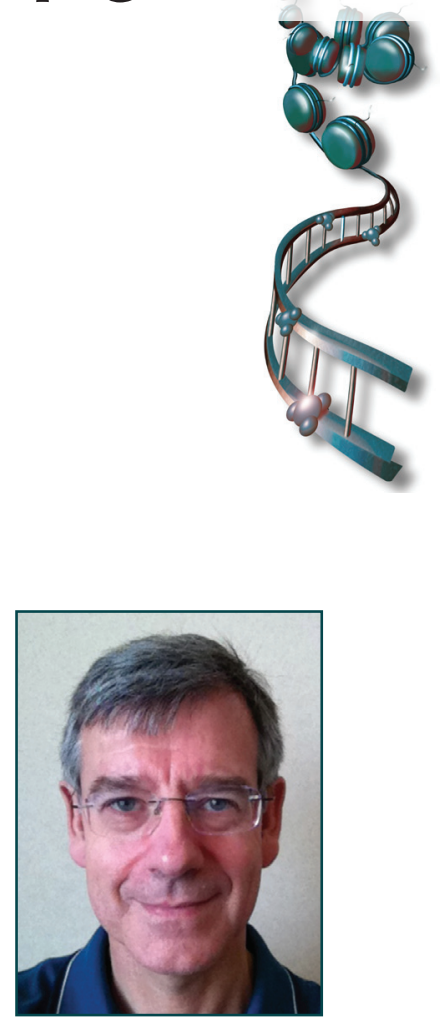

Gavin P Reynolds

Author for correspondence: Biomolecular Research Centre, Sheffield Hallam University, Sheffield, UK Tel.: +44 7740651500 gavin.reynolds@hotmail.com

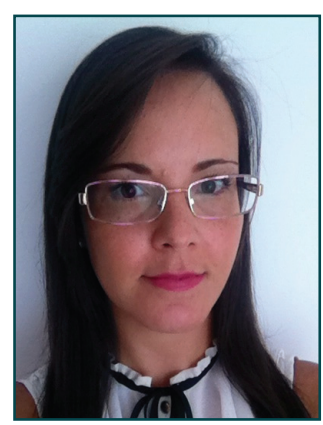

Helene A Fachim

Department of Neuroscience \& Behavior, Medical School of Ribeirao Preto, University of Sao Paulo, Ribeirao Preto, Brazil

and

Institute of Neuroscience \& Behavior - INeC, Ribeirao Preto, Brazil

Future : Medicine part of 
CpG methylation may have multiple influences, both environmental (in the broadest interpretation of that term) and genetic. It is well established that, in addition to genetic variability, environmental factors such as childhood trauma and maltreatment can contribute to an impaired response to antidepressants. Furthermore, such factors can interact with SNPs in their association with response to treatment [1]. This provides a potential indication of, and justification for studies in, epigenetic mechanisms that may link environmental factors to treatment outcome.

Certainly there is increasing evidence that DNA methylation may be involved in the influence of early life stress on depression and antidepressant drug response. As one example, DNA methylation of the serotonin transporter SLC6A4, perhaps the best studied of genes in psychiatric pharmacogenetics, is greater in subjects reporting child abuse, and can influence gene expression by interacting with a common functional promoter SNP, the HTTLPR [2]. Accumulating evidence suggest that this methylation is itself affecting treatment response independent of other influences $[3,4]$. Few other specific genes associated with antidepressant response have been investigated for their methylation status. Interestingly, DNA methyltransferase inhibitors have been reported to have an antidepressant effect in an animal model [5]. It would be valuable to know if this effect extended to human depression, and whether it might address those poorly responding patients with a history of childhood trauma.

\section{"How much this modulation of methylation might contribute to the clinical consequences of drug treatments remains unquantified, but it certainly provides a potential target for therapeutic intervention."}

Of course, the interactions between drugs and a dynamic biological factor such as DNA methylation can be in two directions. Not only can DNA methylation influence the effects of drug treatment, but also psychiatric drugs might modify DNA methylation either globally, or in specific genes, with consequent effects on gene expression that may contribute to drug action. Certainly DNA methylation in blood cells appears to be affected by antipsychotic drug treatment [6]. The major site of action of the antipsychotic drugs is the dopamine D2 receptor; methylation of almost one half of 40 genes related to dopamine neurotransmission is changed following administration of olanzapine to rats, primarily by increased methylation [7]. It seems likely, although not studied, that this might be a common action of all the $\mathrm{D} 2$ receptor antagonist antipsychotic drugs.

While the dopamine system is the target for the effect of antipsychotic drugs on positive symptoms of schizo- phrenia, such as hallucinations and delusions, there is evidence from both pharmacogenetics and clinical pharmacology that the less responsive negative symptoms (e.g., withdrawal, lack of self care) may involve 5-HT neurotransmission [8]. One replicated pharmacogenetics study showed that a functional SNP rs6295 in the 5-HT1A receptor was associated with negative symptom response [9]. A DNA methylation study of the promoter sequence around this SNP, thought to have its effects by affecting transcription factor binding, has identified how methylation of a single $\mathrm{CpG}$ site, itself within a transcription factor recognition site, has a strong correlation with negative symptom response to treatment, paralleling pharmacogenetic findings [10].

Quetiapine, an antipsychotic that also has some efficacy against depressive and manic symptoms, can along with several other mood stabilizers decrease methylation in the promoter region of SLC6A4, perhaps counteracting the SLC6A4 hypermethylation seen in mood disorders [11]. Equivalent results have been obtained from investigation of $B D N F$ promoter methylation in bipolar disease patients undergoing treatment, with similar conclusions being drawn [12].

Understanding the action of valproate is providing clues as to the importance of DNA methylation in psychiatric pharmacotherapy. Valproate is an important drug in the treatment of bipolar disorder as well as epilepsy. Its mechanism of action is not fully understood, although it is an inhibitor of histone deacetylases and induces DNA demethylation, effects that appear to correlate with its ability to relieve symptoms of bipolar illness [13,14]. This results in widespread changes in gene expression, some of which have been proposed as important in symptom relief, including increases in some indicators of GABAergic neuronal activity [13].

Another example of a psychiatric problem that has attracted some interest from epigenetic studies is attention deficit hyperactivity disorder (ADHD). Here too there are established environmental risk factors that may underlie disturbances in DNA methylation, as well as recent evidence for hypermethylation in specific and biologically plausible genes [15,16]. One study has identified how increased promoter methylation in a strong candidate gene - SLC6A3, coding for the dopamine transporter which is a site of action of methylphenidate, the main drug treatment for ADHD - was correlated with poorer response to treatment [Ding et AL., UnPublished].

A concern that runs through all human epigenetic studies of diseases associated with brain dysfunction is the relevance of methylation of DNA from peripheral sources, primarily cells in blood or saliva. Nevertheless there are several studies indicating concordance 
of DNA methylation between these peripheral cells and brain tissue in neurological and psychiatric disease $[17,18]$. Notably a site-specific hypomethylation of the gene for the 5-HT2A receptor, a site of action of newer antipsychotic and antimanic drugs, was found in both brain- and saliva-derived DNA in schizophrenia and bipolar disorder [19].

\section{Conclusion \& future perspective}

Thus accumulating evidence of correlations of clinical outcomes of psychiatric drug treatments with DNA methylation from peripheral samples may well reflect changes in brain DNA. This in turn is likely to result in effects on central gene expression. In this way, DNA methylation changes can be compared with SNPs, although the former is likely to be more subtle while the latter is dichotomous in its effects on gene function. DNA methylation may therefore provide a further measure to enter into personalized medicine algorithms.

In this editorial, we have only cited a few examples of recent research to illustrate the role of DNA methylation in psychiatric pharmacotherapy. Although there are many more valuable studies, work so far has still only scratched the surface of the problem and there are many questions to be answered. Laboratory studies have shown us that DNA methylation can be highly dynamic, and that the extent of methylation in specific sites with potentially functional effects can be differentially modified by a variety of factors including psychological and physiological stress, toxins and psychiatric drugs. The mechanisms underlying these various environmental influences on DNA methylation remain obscure. How much this modulation of methylation

\section{References}

$1 \quad \mathrm{Xu} Z$, Zhang Z, Shi Y et al. Influence and interaction of genetic polymorphisms in the serotonin system and life stress on antidepressant drug response. J. Psychopharmacol. 26(3), 349-359 (2012).

2 Vijayendran M, Beach SRH, Plume JM, Brody GH, Philibert RA. Effects of genotype and child abuse on DNA methylation and gene expression at the serotonin transporter. Front. Psychiatry 3, 55 (2012).

3 Domschke K, Tidow N, Schwarte K et al. Serotonin transporter gene hypomethylation predicts impaired antidepressant treatment response. Int. J. Neuropsychopharmacol. 17(8), 1167-1176 (2014).

4 Kang H-J, Kim J-M, Stewart R et al. Association of SLC6A4 methylation with early adversity, characteristics and outcomes in depression. Prog. Neuropsychopharmacol. Biol. Psychiatry 44, 23-28 (2013).

5 Sales AJ, Biojone C, Terceti MS, Guimarães FS, Gomes MVM, Joca SRL. Antidepressant-like effect induced by systemic and intra-hippocampal administration of DNA might contribute to the clinical consequences of drug treatments remains unquantified, but it certainly provides a potential target for therapeutic intervention. Such an approach is not straightforward; drugs such as valproate that decrease DNA methylation may be unselective in the genes they target and can also be teratogenic, presumably for the same reason.

Although also true for association studies of promoter region SNPs, the intermediate steps that link DNA methylation to drug response and side effects have not been considered in much of this research. Few studies identifying 'pharmacoepigenetic' links have looked further into the mechanisms, which are likely to include effects on transcription factor binding, to assess the role of changes in gene transcription in determining clinical outcomes. A greater understanding of the precise mechanisms whereby changes in DNA methylation lead to different pharmacotherapeutic outcomes, as well as how they interact with functional SNPs, will be invaluable in identifying novel targets that better address the substantial unmet needs in the treatment of major psychiatric disorders.

\section{Financial \& competing interests disclosure}

GP Reynolds has received honoraria for lectures and/or advisory panel membership from Lundbeck, Otsuka, Roche and Sunovion, and a research grant from Sunovion. The authors have no other relevant affiliations or financial involvement with any organization or entity with a financial interest in or financial conflict with the subject matter or materials discussed in the manuscript apart from those disclosed.

No writing assistance was utilized in the production of this manuscript.

methylation inhibitors. Br. J. Pharmacol. 164(6), 1711-1721 (2011).

6 Melas PA, Rogdaki M, Lennartsson A et al. Antidepressant treatment is associated with epigenetic alterations in the promoter of P11 in a genetic model of depression. Int. J. Neuropsychopharmacol. 15(05), 669-679 (2012).

7 Melka MG, Castellani CA, Laufer BI, Rajakumar RN, O’Reilly R, Singh SM. Olanzapine induced DNA methylation changes support the dopamine hypothesis of psychosis. J. Mol. Psychiatry 1(1), 19 (2013).

8 Reynolds GP. The impact of pharmacogenetics on the development and use of antipsychotic drugs. Drug Discov. Today 12(21-22), 953-959 (2007).

9 Reynolds GP, Arranz B, Templeman LA, Fertuzinhos S, San L. Effect of a 5-HT1A receptor gene polymorphism on negative and depressive symptom response to antipsychotic treatment of drug-naïve psychotic patients. Am. J. Psychiatry 163, 1826-1829 (2006).

10 Tang H, Dalton CF, Srisawat U, Zhang ZJ, Reynolds GP. Methylation at a transcription factor-binding site on the 
5-HT1A receptor gene correlates with negative symptom treatment response in first episode schizophrenia. Int. J. Neuropsychopharmacol. 17(4), 645-649 (2014).

11 Sugawara H, Bundo M, Ishigooka J, Iwamoto K, Kato T. Epigenetic regulation of serotonin transporter in psychiatric disorders. J. Genet. Genomics 40 (7), 325-329 (2013).

12 D'Addario C, Dell'Osso B, Palazzo MC et al. Selective DNA methylation of BDNF promoter in bipolar disorder: differences among patients with BDI and BDII. Neuropsychopharmacology 37(7), 1647-1655 (2012).

13 Dong E, Chen Y, Gavin DP, Grayson DR, Guidotti A. Valproate induces DNA demethylation in nuclear extracts from adult mouse brain. Epigenetics 5(8), 730-735 (2010).

14 Phiel CJ, Zhang F, Huang EY, Guenther MG, Lazar MA, Klein PS. Histone deacetylase is a direct target of valproic acid, a potent anticonvulsant, mood stabilizer, and teratogen. J. Biol. Chem. 276(39), 36734-36741 (2001).

$15 \mathrm{Xu}$ Y, Chen X-T, Luo M et al. Multiple epigenetic factors predict the attention deficit/hyperactivity disorder among the Chinese Han children. J. Psychiatr. Res. 64, 40-50 (2015).
16 Wilmot B, Fry R, Smeester L, Musser ED, Mill J, Nigg JT. Methylomic analysis of salivary DNA in childhood ADHD identifies altered DNA methylation in VIPR2. J. Child. Psychol. Psychiatry 57(2), 152-160 (2016).

17 Masliah E, Dumaop W, Galasko D, Desplats P. Distinctive patterns of DNA methylation associated with Parkinson disease: identification of concordant epigenetic changes in brain and peripheral blood leukocytes. Epigenetics 8(10), 1030-1038 (2013).

18 Smith AK, Kilaru V, Klengel T, Mercer KB, Bradley $\mathrm{B}$, Conneely KN et al. DNA extracted from saliva for methylation studies of psychiatric traits: evidence tissue specificity and relatedness to brain. Am. J. Med. Genet. B Neuropsychiatr. Genet. 168B(1), 36-44 (2015).

19 Ghadirivasfi M, Nohesara S, Ahmadkhaniha H-R et al. Hypomethylation of the serotonin receptor type-2A gene (HTR2A) at T102C polymorphic site in DNA derived from the saliva of patients with schizophrenia and bipolar disorder. Am. J. Med. Genet. B Neuropsychiatr. Genet. 156B(5), 536-545 (2011). 